Andreas Kitzig*, Julia Demmer, Tobias Bolten, Edwin Naroska, Gudrun Stockmanns, Reinhard Viga, Anton Grabmaier

\title{
An HMM-based averaging approach for creating mean motion data from a full-body Motion Capture system to support the development of a biomechanical model
}

\author{
Sensor-assisted functionalization of furniture in the hospital and care sector
}

\begin{abstract}
Motion capture systems or MoCap systems are used for game development and in the field of sports for the assessment and digitalization of human movement. Furthermore, MoCap systems are also used in the medical and therapeutic field for the analysis of human movement patterns. As examples gait analysis or examination of the musculoskeletal system and its function should be mentioned. Most application relate to a specific person and their movement or to the comparison of movements of different people. Within the scope of this paper an averaged motion sequence is supposed to be generated from MoCap data in order to be able to use it in the field of biomechanical modeling and simulation. For the averaging of individual movement sequences of different persons a Hidden Markov Model (HMM) based approach is presented.
\end{abstract}

Keywords: Biosignal processing, model driven development, biomechanical modelling, motion pattern database, averaging motion pattern data.

*Corresponding author: Andreas Kitzig: Niederrhein University of Applied Sciences, Faculty of Electrical Engineering and Computer Science, Ambient Intelligence Laboratory, Reinarzstr. 49, 47805 Krefeld, Germany, e-mail: andreas.kitzig@hsnr.de Julia Demmer, Tobias Bolten, Edwin Naroska, Gudrun Stockmanns: Niederrhein University of Applied Sciences, Faculty of Electrical Engineering and Computer Science, Ambient Intelligence Laboratory, Reinarzstr. 49, 47805 Krefeld, Germany, e-mail: \{julia.demmer, tobias.bolten, edwin.naroska, gudrun.stockmanns\}@hsnr.de

Reinhard Viga ${ }^{1}$, Anton Grabmaier ${ }^{1,2}$ : University of DuisburgEssen, Department of Electronic Components and Circuits ${ }^{1}$, Bismarkstr. 81, 47057 Duisburg, Germany,

Fraunhofer Institute for Microelectronic Circuits and Systems $\left(\right.$ IMS) ${ }^{2}$, Finkenstraße 61, 47057 Duisburg, Germany, e-mail: reinhard.viga@uni-due.de, anton.grabmaier@ims.fraunhofer.de https://doi.org/10.1515/cdbme-2018-0093

\section{Introduction}

In the field of MoCap systems, a great deal of progress has been made in terms of precision and reliability in the recent years. The systems were originally intended for use in computer game development, however, there are more and more applications in the medical and diagnostic field. In this area, MoCap systems are e.g. used for gait analysis [1] or motion strategy analysis [2]. In sports, MoCap systems are used, for movement analysis e.g. of karate fighters [3]. The referenced work also presents an averaging procedure [3], which, focuses on the averaging of a very precisely executed movement sequence of a person. We are currently working on a biomechanical model for the model-driven development of functional nursing beds in the clinic and care sector. The basic model has already been presented in [4].

A big challenge here was that the movement sequences of humans in the nursing bed could not be adequately modeled. The use of data from a markerless full-body MoCap system (MoveHN [5]) empowered us to utilize human movement patterns in our modeling approach, but an average movement pattern for general test series was lacking. A procedure for averaging the movement data of the MoveHN database has already been presented in [6] but the suggested approach has proved to be unreliable. For this reason, a new method for creating average movement sequences out of data from the MoveHN database based on Hidden Markov Models (HMM) is presented. 


\section{Database MoveHN}

In order to be able to carry out own experiments and to be able to use appropriate data for the modelling of bodyposture and -movement the database "MoveHN" was created, which contains movement patterns of real humans. The XSens MVN Awinda System [7] was used for this purpose. The system consists of cordless trackers attached to the human body to derive the motion of the person who is wearing the measuring devices. The recorded movements are provided in the form of a stickman model for further tasks. The focus of our dataset acquisition is on movement sequences and body positions of people in nursing beds. Therefore, corresponding movement sequences were defined. A total of 680 files, which cover about 400 minutes of motion data were recorded. The acquired data originates from five male and five female test persons, each of which run through two recording sessions on two different days. A complete overview of the MoveHN database can be found in [5]. MoveHN can be downloaded at: http://ami.kr.hsniederrhein.de/moveHN

\section{HMM averaging}

As already mentioned in the introduction, the approach presented in [6] for generating an averaged movement sequence from the XSens data was not sufficient. This was due to the fact that the averaging for the same data set resulted in different sequences of movements depending on the input sequence. Furthermore, the approach was not very flexible regarding the adjustable parameters.

To overcome this drawback, the current approach uses parts of a Hidden Markov Model (HMM) based pattern recognition system. HMMs are well known from speech recognition but in our case the HMM can be useful as well because HMMs can also be used as a kind of generator. In this work this option was used to generate average motion data. The HMM itself contains average information about the process to be modelled as well as corresponding information regarding the temporal structure of the process. In addition, a large number of parameters can be set (number of states and Gaussian distribution, level of detail of HMMs, etc.). The individual steps for averaging the movement sequences will be described in detail in the following section.

A schematic of the approach is shown in Figure 1. The pre-processing and re-calculation is done with Mathworks Matlab, the calculation of the HMMs is done with HTK [8].

\subsection{Generation of HMMs from XSens motion data}

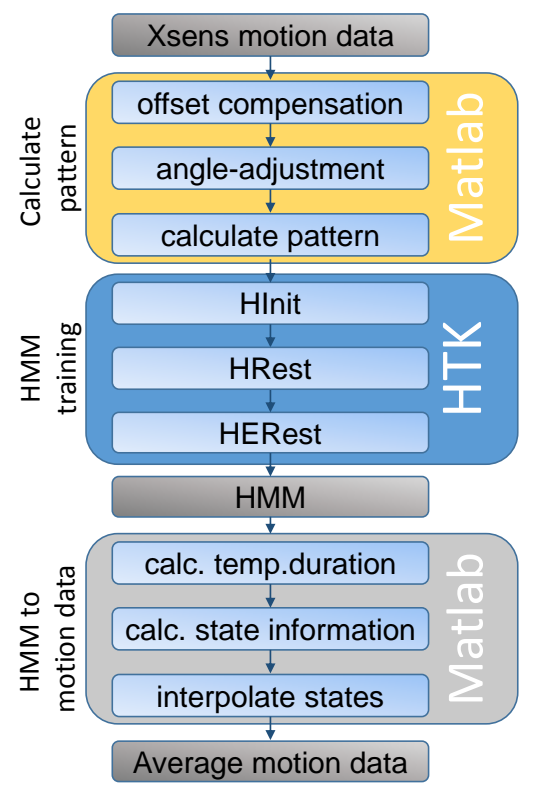

Figure 1: Schematic of the motion data averaging system

In order to prepare the patterns for the generation of HMMs, a pre-processing of the Xsens data for normalizing the positions through offset compensation and angle-adjustment is necessary. The offset compensation is used to center the datasets of the individual persons in the area of the pelvis for the respective action in the zero point of a three-dimensional Cartesian coordinate system by means of coordinate shifting. In the next step, the rotation of the body in the $x-y$ plane is calculated and adjusted accordingly so that the head and pelvis alignment is along the $\mathrm{x}$-axis. These steps are necessary because the individual data records already differ greatly in relation to zero position and alignment in the basic data. Following this, the $\mathrm{x}, \mathrm{y}$, and $\mathrm{z}$ position coordinates (representation of length, width and height in relation to the reclining area) for each body part are stored as feature vectors of 63 elements for each sample in chronological order as a pattern. Then the generation or "training" of the HMMs starts with "HInit". That function performs two basic steps to create an initial HMM from the input patterns. In the first step, a "bootstrapping" is performed in which all the associated segments for a specific action are read initially. Then each segment is split linearly into subsections. The number of subsections depends on the number of emitting states of the chosen HMM structure. Furthermore, the mean vector and the covariance matrix are estimated from all subsections. This is followed by a Viterbi training, in which the boundaries between the subsections across the segments 
are optimized by a Viterbi aligner. Furthermore, there is a reestimation of the mean vectors and covariance matrices of the new subsections. These steps are repeated until the total probability of all states of the model converges or a maximum number of iterations is reached. Convergence is achieved when the probability values no longer change to the previous iteration step. After the execution of "HInit", a segmental Baum-Welch training is performed in "HRest" to re-estimate the parameters of the initial HMMs. By this processing step, an improvement of the later HMMs is achieved. Beside the HMMs also the label information and pattern files are used in "HRest". The Baum-Welch training takes all possible paths through the HMM into account using a recursive estimating algorithm to create robust parameters in the Gaussian mixtures and in the transition probabilities of the HMM. A final refinement of the HMMs is achieved by "HERest". The label information is no longer used and an embedded training takes place in which a single BaumWelch estimate of the entire HMM set is performed simultaneously. This can be compared to a kind of "forced" recognition in which the attempt is made to map the already created models to the respective basic data. After these processing steps each resulting HMM represents one averaged motion sequence which can then be further processed.

\subsection{Calculation of motion data from HMMs}

After a linear HMM structure without state skips is generated, the temporal structure of the respective HMM is estimated. Here an adapted approach from [9] is used. First, the duration of staying in a state $S_{n}$ can be determined from the conditional probability $p\left(S_{t}=S_{n} \mid S_{t-1}=S_{n}\right)$ :

$$
\mathrm{d}\left(S_{n}\right)=\frac{1}{1-p\left(S_{t}=S_{n} \mid S_{t-1}=S_{n}\right)} \cdot t_{\text {shift }}
$$

Here $t_{\text {shift }}$ represents the overlap of the feature vectors. Subsequently, the time $\mathrm{t}\left(S_{i}\right)$ for the occurrence of a state $S_{i}$ can be calculated by assuming the center of the time interval of the associated state.

$$
\mathrm{t}\left(S_{i}\right)=\sum_{j=1}^{i-1} d\left(S_{j}\right)+\frac{d\left(S_{i}\right)}{2}
$$

After the temporal information of an HMM has been determined, the corresponding parameters, which are

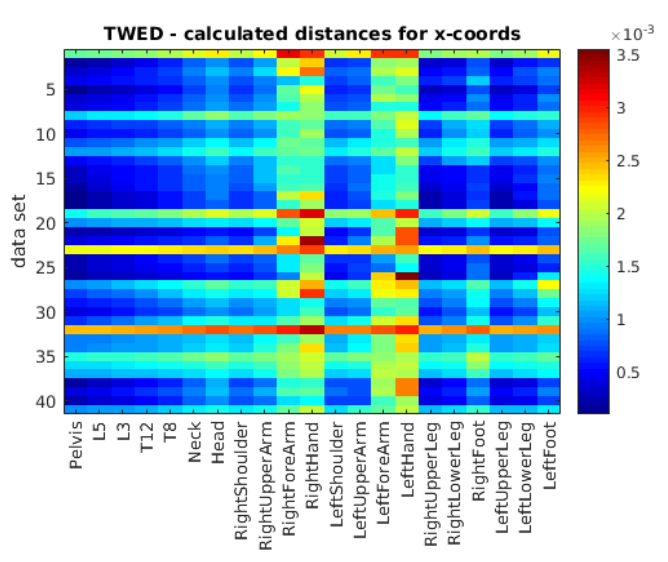

Figure 2: Distances for HMM and the datasets, $x$-axis

described by a multi-dimensional distribution density function and modelled in the states are calculated.

In this work, Gaussian distributions are used so that the function can be described by mean value and variance. For the coordinate features contained in the HMM, the number $\boldsymbol{N} \boldsymbol{R}_{-} \boldsymbol{m i x}$ of Gaussian distributions $\boldsymbol{c}_{\boldsymbol{k}}$ are summed and multiplied by the mixture weighting factor $\boldsymbol{w}$ in the HMM.

$$
X_{k}=\sum_{j=1}^{N R \_m i x} w\left(m i x_{j}\right) \cdot c_{k}\left(m i x_{j}\right)
$$

The resulting values are finally brought into a suitable temporal structure by means of spline interpolation. This happens by taking the corresponding sampling frequency into account.

$$
\begin{gathered}
\left\{X_{k}\left(t\left(S_{1}\right)\right), X_{k}\left(t\left(S_{2}\right)\right), X_{k}\left(t\left(S_{3}\right)\right), \ldots\right\} \\
\stackrel{\text { Spline }}{\longrightarrow}\left\{X_{k}(0), X_{k}\left(t_{\text {shift }}\right), X_{k}\left(2 \cdot t_{\text {shift }}\right), \ldots\right\}
\end{gathered}
$$

The final values correspond to the coordinates and their change of an averaged motion sequence and can either be visualized or applied in the biomechanical model.

\section{Results}

From the data of the MoveHN database different averaged movement squences were created. The parameter of the generated HMMs are shown in Table 1. 
An HMM-based averaging approach for creating mean motion data from a full-body Motion Capture system to support the development of a biomechanical model -392

Table 1: HMM averaging parameter.

\begin{tabular}{lr}
\hline Parameter & Value \\
\hline Number of motion sequences/HMMs & 17 \\
Number of states in each HMM & $20 / 18 / 2$ \\
(total/emitting/dummy) & \\
Number of Gaussian Mixtures per state & 1 \\
Sample rate $\boldsymbol{f}_{\boldsymbol{s}}$ of input data & $60 \mathrm{~Hz}$ \\
$\boldsymbol{t}_{\text {shift }}$ & $\mathbf{1} / \boldsymbol{f}_{\boldsymbol{s}}$ \\
\hline
\end{tabular}

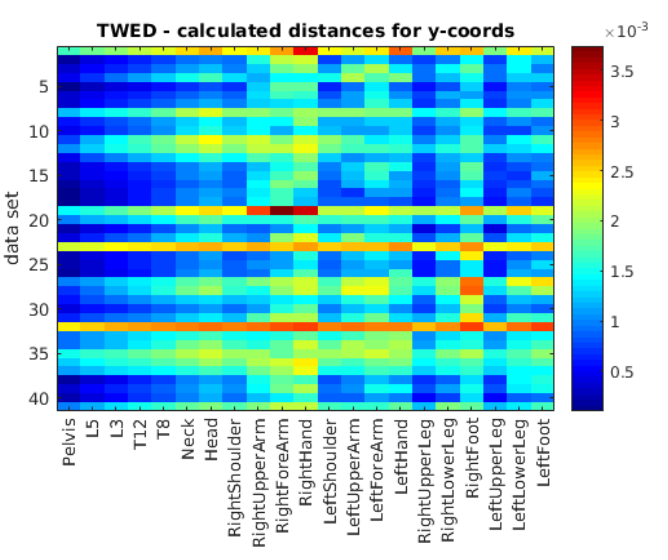

Figure 3: Distances for HMM and the datasets, $y$-axis

The resulting averaged data sets were initially visually rated, which is good as a criterion for a first review but is very subjective for the rating. In order to be able to decide to what extent the basic data for the different humans and recording sessions differed from the respective averaged movement sequence, the Time Warp Edit distance (TWED [10]) was determined as distance measure. As an example, the distance for the movement "turn back to front", a turn from the supine position to the prone position, was calculated. The individual distances are shown in so called heat maps in the figures 2 to 4 separately for every body part (L5, L3, T12 and T8 represents the spine) for 41 different data sets. In the individual figures it can be clearly seen that the biggest differences are in the area of arms and hands. This is due to the fact that the persons were free in the choice of individual movements when changing positions. Furthermore, it can be stated that there are generally larger deviations in the area of the $\mathrm{x}$-axis and the $\mathrm{z}$-axis. This is also due to the variability of the same motion sequence.

\section{Conclusion and outlook}

The paper presents a method for creating an averaged motion sequence from MoCap data based on HMMs. It could be shown that the method works well and produces reliable average movement sequence results. From the results of the comparisons of the individual data sets with the averaged results, it becomes clear that deviations occur, especially in the area of hands and arms. These could also show up in the averaged movement sequences and lead to a disruption of the sequence of movements in the multi-stage model.

As a compensatory measure, a subsequent filtering of the movement patterns and normalization of body size of persons is envisaged.

\section{Author Statement}

Research funding: The author state no funding involved. Conflict of interest: Authors state no conflict of interest. Informed consent: Informed consent has been obtained from all individuals included in this study.

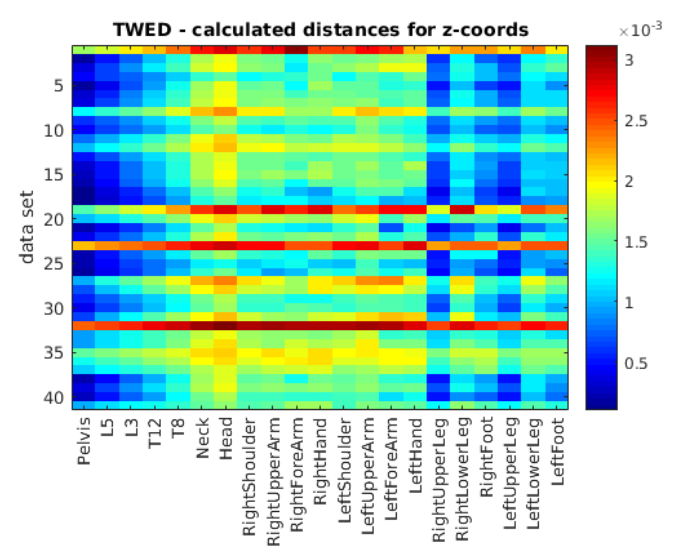

Figure 4: Distances for HMM and the datasets, z-axis

\section{References}

[1] Loose, H.; Orlowski, K.: Gait patterns in standard scenarios: Using Xsens MTw inertial measurement units, 16th International Conference on Research and Education in Mechatronics (REM), Bochum, 2015

[2] Burget, F.; Maurer, C.; Burgard W.; Bennewitz, M.: Learning motor control parameters for motion strategy analysis of Parkinson's disease patients, IEEE/RSJ International Conference on Intelligent Robots and Systems, 2015

[3] Hachaj, T.; Ogiela, M. R.; Piekarczyk, M.; Koptyra, K.: Averaging Three-Dimensional Time-Varying Sequences of Rotations: Application to Preprocessing of Motion Capture Data. In: Sharma P., Bianchi F. (eds) Image Analysis, SCIA 2017. Lecture Notes in Computer Science, vol 10269. Springer, Cham.

[4] Kitzig, A.; Naroska, E.; Stockmanns, G.; Viga, R.; Grabmaier, A.: A novel approach to creating artificial training and test data for an HMM based posture recognition system, IEEE 26th International Workshop on Machine Learning for Signal Processing (MLSP), Vietri sul Mare, pp. 1-6. 2016

[5] Kitzig, A.; Schröter, S.; Naroska, E.; Stockmanns, G.; Viga, R.; Grabmaier, A,: MoveHN - A database to support the 
An HMM-based averaging approach for creating mean motion data from a full-body Motion Capture system to support the development of a biomechanical model -393

development of motion based biosignal processing systems, 25th European Signal Processing Conference, 2017

[6] Kitzig, A.; Schröter, S.; Naroska, E.; Stockmanns, G.; Viga, R.; Grabmaier, A.: Improvement of a multi-stage model for the modeling of a functionalized nursing bed as support for the sensor-assisted functionalization of furniture in the hospital and care sector In: Current directions in biomedical engineering Jg. 3 (2017)

[7] XSens-Document MV0319P, User Guide MVN, MVN BIOMECH MVN Link, MVN Awinda, Revision P, Oct. 2015

[8] Young, S. J. : "The HTK Hidden Markov Model Toolkit: Design and Philosophy", Entropic Cambridge Research Laboratory, Ltd, 1994

[9] Hirsch, H.-G.; Kitzig, A.: Visualisierung der in einem HMM enthaltenen spektralen Merkmale, 9. ITG Fachtagung Sprachkommunikation, 2008

[10] Marteau, P.-F.: Time Warp Edit Distance with Stiffness Adjustment for Time Series Matching. In: IEEE Transactions on Pattern Analysis and Machine Intelligence. Band 31, 2009 